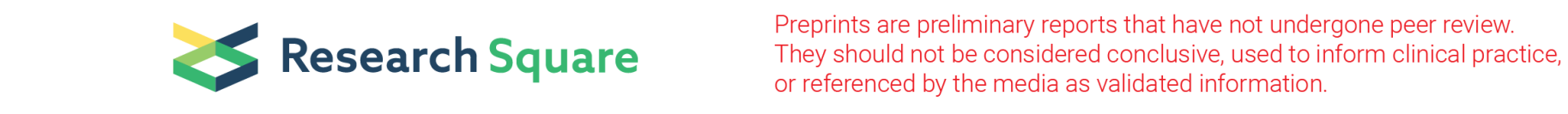

\title{
DDoS Detection and Prevention of Attacks on M-Health Sensitive Data: A novel approach
}

\author{
Soumya Ray \\ BIT Mesra: Birla Institute of Technology \\ kamtanath mishra ( $\sim$ mishrakn@yahoo.com) \\ Birla Institute of Technology, Ranchi

\section{Sandip Dutta} \\ BIT Mesra: Birla Institute of Technology
}

\section{Research Article}

Keywords: m-health, security and privacy, DDoS detection, DDoS prevention

Posted Date: January 19th, 2022

DOl: https://doi.org/10.21203/rs.3.rs-800163/v1

License: (c) (i) This work is licensed under a Creative Commons Attribution 4.0 International License. Read Full License 


\section{Abstract}

In today's world m-Health (also termed as Mobile Health) is an integral part of the healthcare industry. M-health uses various kinds of mobile devices such as mobile phones, personal digital assistants, tablets, laptops, and wireless-based arrangements to collect and transfer run-time medical data between patients and healthcare institutions. The entire process of the m-healthcare system is dependent on a cloud system. As a result of services provided through the cloud platform, it is observed that network equipment is likely prone to different types of attacks in the system. The most extensively used of these attacks are Distributed Denial of Services (DDoS) attack which can halt the network service instantly and prevent the accessing of sensitive data. The attack can destroy the privacy and security of the data by injecting wrong information into it. The most crucial way in the battle against DDoS attacks is the fast detection and taking apart of network traffic. In this research work, four layers of $\mathrm{m}$-healthcare architecture and the specific reasons for DDoS attacks in the system architecture are explained properly. A novel DDoS detection algorithm is proposed for the early detection of the attack in the system. The DDoS preventive algorithms are designed efficiently to restrict the access of attackers in the system. Further, the effect of the different types of DDoS attacks with their preventive approaches experimented in the cloud-based simulation environment. The maximum success and average success of the attacker concerning time are also analyzed as a part of the research paper.

\section{Introduction}

Mobile health ( $\mathrm{m}$-health) is a swiftly growing field in the digitized healthcare domain. This booming technology provides faster access to healthcare support to ailing patients, easy communication to the medical personnel using mobile-based technologies such as tablets, smartphones, and wearables. M-health technologies allow patients to deliver secure messages through text, emails, video calling, and voice calling rapidly, schedule appointments with the doctor, and connect the providers 24/7 for telemedicine support. This technology permits access to electronic health records (EHR) of a patient and clinical decision support tools to the authorized medical person. The decision support tools are the huge amount of digital data through which patients' medical conditions can be judged [1-4].

Healthcare system uses different kinds of heterogeneous data to provide medical services irrespective of the time and location of the patient and medical personnel. An aged person can check his blood pressure and send the report to the doctor using mobile devices. Chronic heart disease can be treated if the patient is far away from his point of the nursing station. The patient can collect the blood glucose information from the wearable devices and send it to the doctor for the interpretation of his current health status [5,6]. Diagnosis results, type of medicine prescribed to an individual patient, time of giving medicine, and dose of medicine are the important sensitive attributes of a patient's healthcare information. Apart from this medical data healthcare domain certainly handles statistical data, financial data, and insurance-related data. These are very sensitive data to a patient as well as the employees engaged in their different organizations. Loss of data or hacking of data by an intruder can damage the privacy of the patient. Whenever the data passes through different internetworking systems proper security safeguards are mandatory to protect the sensitive data [7-10].

To access sensitive medical data smart phones, play a major role to manage the various needs of stakeholders. Using a smartphone medical person can view patient data, store it for future reference, and can share it for another opinion. In the same way, the patient can use it to access, update, and control their medical information. Apart from this few applicationbased software's like "Stress check', "Lose it" and "ZocDoc" are available to collect and transfer information between different stakeholders. The non-medical sensitive information can also be passed through this different $\mathrm{m}$-health application software [11-14].

The major challenge of this $m$-health technology is the security assurance of medical and other sensitive data. Data availability is the most distressing security issue among them. The most vulnerable distributed denial of service (DDoS) attack hampers the availability of patient data continuously as well as hinders the flowing of data across the internetworking system $[15,16]$. The major objective of a DDoS attack is not only to breaching of data, it can restrict the user 
to access necessary information from a portal also. The Cybersecurity \& Infrastructure Security Agency (CISA)claims that identification of DDoS attacks is very tricky as it can be performed virtually. The companies used to handle different kinds of sensitive data are the major targets of this attack. The sensitive data can be modified by malicious intruders and false information may be injected into the different data streams by a false node [17,18]. A medical record can contain a higher significant financial value associated with credit card data. So, proper DDoS detection and prevention mechanisms are necessary to tackle the issues related to $\mathrm{m}$-healthcare. Access control mechanisms like authentication, authorization, identification and accountability are highly needed to protect sensitive data. Apart from these security and privacy issues the technology also suffers from the low bandwidth of the mobile network, high cost for cellular network links, inadequate availability of internet connections, and the heterogeneous platforms supported by different mobile devices. These problems must be addressed properly to develop a complete security solution of this technology with easy accessibility in real-life scenarios [19-22].

Figure 1 represents a sensitive data access technique in the healthcare domain. The request for sensitive data access may be generated from the user level (through the mobile, desktop, or any Healthcare Related Organisation) and passed to the cloud healthcare system. Next, it redirects the request to the authentication server through API (Application Programming Interface) call which maintains the Auth Key for the valid user. The authentication server sends the response back to the cloud with the Auth key. Together with Auth Key, an access request will go to the Data server where Sensitive data is stored. Access control server generates the Auth key and corresponding Access key. Thereafter it is sent to the Authentication Server as well as the sensitive data server. Access control server can revoke the permission of the key in a time-bound process from both the server. If the Auth key of the request is valid during that period then sensitive data is ready for access with that key. The user will be able to access it only if he has the corresponding private key of the access key received from the sensitive data.

The entire process of sensitive data access techniques in $\mathrm{m}$-healthcare is prone to vulnerable DDoS attacks. This will significantly hamper patient care as well as accessing sensitive information, files, records, and emails from the cloud-based system. The major contribution towards this research paper is described below.

- The research paper concentrates on the significant effect of DDoS attacks in the m-healthcare system. The authors have presented $\mathrm{m}$-healthcare architecture along with the possible reasons for this vulnerable attack in the cloud-loTbased system.

- The authors have provided a novel DDoS detection algorithm for easy identification of the attack in the system.

- Different kinds of DDoS attacks and their preventive approaches are explained and analyzed.

- Finally, the significant effect of different kinds of DDoS attacks along with their prevention mechanisms is experimented and analyzed with the help of a cloud-based software environment.

The remaining paper is organized as follows. Section 2 introduces the related work. Section 3 presents the architecture of the $\mathrm{m}$-health system. Section 4 highlights the DDoS detection algorithm. Section 5 explains the prevention mechanism of DDoS attacks in the m-healthcare system. Simulation results and evaluation are explained in Sect. 6. Finally, we conclude our research work in Sect. 7.

\section{Related Work}

Security and privacy in the $\mathrm{m}$-healthcare system need a major consideration as it handles the different sensitive and private information of a patient. The sensitive health-related information is gathered and passed through resource constraint mobile nodes over the wireless medium to the health data canter. Thereafter authorized medical professionals can analyze the patient's records for proper diagnosis and better treatment. However, due to the passing of sensitive information through insecure wireless network medium, it is often prone to different attacks which can alter the original information or inject false information. This leads to a wrong diagnosis as well as hampers the proper treatment of a patient. The objective of 
the paper is to bring attention to the most vulnerable DDoS attack and its consequences in the m-healthcare system. Several researchers are carried out their researches to overcome the problem and to provide a unique solution [23-25].

Aditya Rajagopalan et. al., [26] have explained a DDoS prevention scheme for security in loT-based healthcare systems. The model helps to prevent DDoS and replay attacks as all the requests to the sensors are passed through the e-Health Gateway smart system of the fog layer. S. Rahimi Moosavi et. al., [27] stated that health data related to a patient is the most sensitive information and can't be disclosed publicly. Secure end-to-end communication between the end-users and the m-health system is mandatory to contain DDoS or any other vulnerable attacks. Their observation indicates that the distributed communication between the m-health system is provided by the e-Health Gateway which is supported by Datagram Transport Layer Security protocol (DTLS).

Prajakta Kamble et al., [28] have proposed machine learning and deep learning algorithms to improve the security of loTbased systems. The algorithms are used with the different encryption methodologies for secure communication between the user and the m-healthcare system. The encrypted algorithms with the proper identity authentication help to mitigate the DDoS attack and intrusion detection in the system architecture.Ziegeldorf. J. H. et al., [29] have presented the different vulnerable attacks in the loT-based $\mathrm{m}$-healthcare system. The objective of the research is to provide more focus to the upgradation of the existing framework and maintain DDoS attack detection and prevention strategies.

Confidentiality, integrity, and availability of patient data are the major concern in the loT-based healthcare system. The healthcare system must be equipped with a resilient structure along with optimum security performance. A. M. Rahmani [30] has stated that the devices involved in the healthcare system must be trained to detect the attacks. In the loT-based system, attacks can be originated from the inter/intra networking system. They can easily take the control of the system and the sensitive information related to the patient can easily be revealed or tampered with. The controlling nodes are very muck attack prone in the system. S. Sivagami et al., [31] have suggested a secure routing protocol is necessary at the time of communication between different nodes.

J.Jhang et al., [32] have presented the efficiency of blockchain technology to store and passing of data securely in a distributed environment. The blockchain is successfully introduced in many sectors (e.g., healthcare, financial) to accomplish a very advanced level of transparency and reliability in the network. In [33] the development of a new protocol DOTS (DDoS Open Threat Signalling) has been proposed by the Internet Engineering Task Force. The protocol is comprised of a DOTS client and a DOTS controller. As soon as the DOTS protocol detects an attack it will inform the client and call the mitigation service through the controller. The controller is responsible to make coordinating between the different components in the networking system. In [34] Steinberger et al., have explained the uniqueness of the DOTS protocol. The authors have used a dynamic flow-based message exchange methodology to ease the collaboration and deployment between internetworking domains.

K.Giotis et al., [35] have presented the new collaborative DDoS attack prevention scheme with the help of $B G P$ (Border Gateway Protocol). Any modification to the protocol is very difficult and the latency of the protocol report generation may be considerable. The major obstacle of this scheme is that reports can be spoofed and generated from unauthenticated domains too. In [36] G. Zhang et al. have stated a "gossip-based approach" to detect and pass the attack information to the neighboring nodes. They have designed a model based on a peer-to-peer network for the rapid spreading of attack information. Bahman et al., [37] have suggested a new DDoS attack prevention scheme "CoFence" among the multiple network virtualized function domains. As soon as any particular domain faces an attack then it redirects the entire traffic to the other domain for the filtration of the illegitimate packets. This model is significant to identify the threats at the time of passing sensitive data to the different nodes in the system.

\section{The Architecture Of M-healthcare System Model}


In this section, the authors have explained the four-layer mobile healthcare architecture system. The architecture supports collaborative and distributive data management along with three major criteria. First of all, data transfer and data accessing will be more optimized for localized data. Distributive and direct data processing at any stage of the healthcare system provides low response time and the workload between different nodes will be minimal. The overall interfaces should be designed in such a way that user-level experiences at the time of registering and accessing the system are satisfactorily improved. The model can be extended to maintain healthcare data for users fit in different healthcare services, e.g., private clinics and government hospitals. The primary objective of the proposed healthcare system is to collaborate with the different network entities for better data management. The detailed specification of network-based entities is specified below.

\subsection{User Layer:}

The m-healthcare system provides various application software and a huge amount of data is generated at the time of providing services to different users. Users are also using different healthcare service providers in their systems. The major purpose of this layer is to collect healthcare data from different heterogeneous wearable devices (e.g., smart gloves, smart wriest devices) and sent it to the clinics, hospitals, and any medical institution by authorized medical professionals. These EMRs (Electronic Medical Records) are stored in the respective institutions for immediate treatment of critically ill patients. The data is further uploaded to the central cloud system by the authorized person so that it can be accessed from anywhere with a valid identity. The healthcare data must be passed through the secure data transmission medium with proper identity authentication techniques. It is observed that data accessing is prevented on time due to different attacks in the system. The paper mainly focuses on DDoS attacks in the system and how does this attack creates major trouble at the time of passing run time healthcare data from one network to another network in the system architecture.

\section{2 faceless interaction layer:}

This layer is one of the most important components of the m-healthcare system. The layer supports accessing of data and responds to patient's requests through many channels irrespective of the device or location. It provides necessary data to the healthcare professionals that meet their needs. Patients can get an improved experience with a self-service option to access healthcare records by a simple interaction medium in real-time. The entire communication between patients and medical professionals is done through a multi-channel integration networking system.

\subsection{Application layer:}

The application layer controls a large amount of healthcare data in a collaborative way among the different network edges. This process is beneficial for managing workloads among the different nodes in the system. The application layer is useful to reduce delay at the time of processing a bulk amount of healthcare data. The fundamental functionalities of this layer are given as follows.

\subsubsection{User registration:}

The registration to the $\mathrm{m}$-health system is done in this layer. Users can register themselves with valid identities. After the successful registration trusted authority of the healthcare system will provide the public/private key pair and digital signatures to them. Thereafter users can access information from the remote cloud system.

\subsubsection{Data Storage:}

The application layer stores raw heterogeneous healthcare data in an encrypted format. The past information related to data processing, storing, and accessing is recorded in an ordered fashion. This process will effectively reduce the information searching time from the massive amount of data. The system also ensures to find the leakage of information that happened due to security attacks.

\subsubsection{Data processing and Analysis of m-healthcare data:}


Processing raw healthcare data is the most important task after successful storing of data. Different formats of data are stored in the healthcare domain. So, robust, complex approaches related to cleaning, reduction, integrated and normalized approaches are implemented. The application layer also supports of non-revealing of sensitive data to unauthorized users.

The healthcare service providers are capable of processing large amounts of healthcare data dynamically. They support real-time as well as an off-line mode of processing based on a different scenario. Data processing is done quickly for a patient admitted to a critical care unit. The summarized data analysis report is sent directly to the medical professionals for low latency response. Apart from the services given to the critical care unit patient, the medical recommendation to the patients and off-line analysis of data is the important functionality of this layer.

\subsection{Central Layer:}

The central layer works as a top security manager and is held responsible for the security protection of different components of the $\mathrm{m}$-healthcare system. The entire data management operation is performed in this layer. This layer stores and accesses all user-specific information with its highest priority. The mapping relationship among the different user's data is also stored in this layer. The central layer employs a global database to maintain and update a large amount of healthcare information. The major functionality of this layer is to provide access control to the user based on the identity authorization technique. Revocation of access control operation is performed in special cases, e.g. if a user is found doing malicious activity in the system and the security of the system is compromised.

\section{The Possible Ddos Attack In M-healthcare:}

The distributed denial of service (DDoS) attack is used to interrupt the target server, network, and services by sending a huge number of repetitive requests. This will generate a flood of internet traffic with networks of interconnected machines. The network consists of different kinds of heterogeneous systems (IoT-based devices) which are attacked and controlled by the attacker virtually. The individual devices are called bots and the attacker sends instructions to each bot to maline the system resources. The bot is used to send false information to the server IP addresses repeatedly to overwhelm the network and creates a DDoS attack. The major DDoS attacks in m-healthcare are Application-level attacks; HTTP flood attacks; SYN flood attacks; DNS flood attacks.

The application layer DDoS attack is designed to attack the application running in the system. This attack prevents to deliver the sensitive data on time at the proper destination. The low to mid-volume level of this attack is initiated by intelligent clients or loT-based devices. The attack has a significant drawback in the m-healthcare system. When a medical professional is trying to search the patient history from the application server/remote cloud server he has to provide its login credentials to the system. It is observed that during the checking of login credentials the resource consumption is high and loading of relevant data from the database is time-consuming. This ultimately maximizes the request-response generation time. In the HTTP flood attack, the attacker sends HTTP requests continuously to make the webserver overloaded. As soon as the server reaches its maximum amount of request handling capability, then no further request is accepted. The situation leads to a DDoS attack for the normal user. This attack will prevent the run time medical data to the destination on time. As a result, the online/offline mode of data analysis will not be functional and the generation of EMR for critically ill patients will be delayed.

The SYN flood attack is designed to target any systems connected to the internet and providing services (e.g., file server, email server) through TCP. The attackers consume the active connection states of different infrastructure components including firewall, intrusion detection system, and the remote web server also. This attack slows down the high-capability devices which are connected to multiple connections. The attack will prevent the passing of runtime medical data between different ports of the inter/intra networking system. In the case of a DNS attack, the Domain Name System (DNS) is used by the internet-enabled devices through which they can look up the web server for the access of internet content. The attacker floods the server of a particular domain to prevent the DNS resolution for the specific domain. This will lead to the 
application server, API, website become unresponsive to the legitimate traffic. As a result, patients, doctors, and medical staffs are not able to access the website of the hospitals. Request and response generation will completely be destroyed. This is a significant drawback of DDoS attacks in the mobile healthcare system.

\subsection{Necessary conditions for DDoS attacks}

The main objective of a DDoS attack is to make it impossible for a service to be delivered. This is possible by the attackers who will access the servers, databases, services, network applications virtually. In general, the attacks will work by drowning the system with voluminous data requests. The legitimate users will send requests from different IP addresses and ultimately it consumes huge network bandwidth, memory, and CPU power. This will ultimately crash the system under the demanding situation. Due to this attack, the patients and the medical professionals will not able to communicate or pass data between them. The server is blocked by huge data requests. So, request and response generation are affected by the attack. The possible necessary conditions of the DDoS attacks are explained below.

\subsubsection{Degradation of Network Performance:}

DDOs attack slows down the performance of the network. But it will be difficult to check whether the traffic is coming from one IP or multiple sources. The exact purpose of a DDoS attack is to have traffic generated from multiple IP addresses. The slow opening of files and unavailability of website resources are the results of DDoS attacks.

\subsubsection{Unavailability of the particular website:}

It is observed that sometimes the website is down in a peak time where maximum numbers of hits are generated. This can be possible for a particular time frame. But if this unavailability of the website continues for a day or more then there is a possibility of DDOs attack in the system.

\subsubsection{High increase in the number of spam emails:}

A high increase in the number of spam emails causes a significant hamper by infecting user's systems with malicious software. This ultimately damages the system and steals personal information. The situation will lead to huge network resource consumption and bandwidth in the system.

\subsubsection{Repeated request for same data:}

To perform the internal operations of the website sending and receiving data is mandatory. If the same data is requested multiple times from the same source, then there may be a chance of attack in the system. This attack will prevent reaching the data to the destination on time. But this process will consume huge network bandwidth too.

Fig.3 presents a layered interaction model of the m-healthcare system. All four layers are connected and integrated. The coordination of request and response generation is maintained by the integration of four layers. The $\mathrm{m}$-health system is more prone to DDoS attacks. All the necessary conditions of this vulnerable attack are depicted in the diagram. As soon as the DDoS attack is found, the system will take the necessary preventive mechanism to maintain the smooth functioning of the workflow.

\subsection{TheProposed DDoS Detection Algorithm (PDDA)}

In the cloud network, the distributed end nodes are engaged in detecting the DDoS attack. The proposed DDoS detection Algorithm 1 of Fig. [4] checks the incoming traffic at regular intervals in the cloud network. The traffic may be normal or it may fall under DDoS attack. The algorithm will specify the attack type and set an alert (alert may be two types: high or low) as soon as DDoS traffic happens in the system. It will send the alert message with the attacker's Internet Protocol address 
(IP), port number (source, destination), and also attack type for monitoring purposes. The following part provides the complete PDDA algorithm.

The proposed detection algorithm creates a table to check the status of the incoming packets received from the various clients during the fixed time interval. The said time deviation is called detection time. The table contains the following field information: Internet Protocol Address of the sender, Source and Destination Port number individually, Counter of received packets, and the time gap between the first and last packet. The data will be inserted for the above-mentioned fields.

Thereafter the execution of the algorithm will check the data value and update the alert type accordingly. The PDDA table considers the three scenarios to define the structure of the table. The first case is where the source port and destination port number are the same. The second case highlights the same source port but the changes of destination port number. The third case observes the changes of source port numbers maintaining the same destination port. Table [1] represents the PDDA table structure format.

Table 1

PDDA table structure format

\begin{tabular}{|lllllll|}
\hline $\begin{array}{l}\text { SI. } \\
\text { No. }\end{array}$ & $\begin{array}{l}\text { Internet Protocol } \\
\text { Address }\end{array}$ & $\begin{array}{l}\text { Port Number } \\
\text { (source) }\end{array}$ & $\begin{array}{l}\text { Port Number } \\
\text { (Destination) }\end{array}$ & $\begin{array}{l}\text { Counter of } \\
\text { Packet }\end{array}$ & $\begin{array}{l}\text { Time } \\
\text { Difference }\end{array}$ & $\begin{array}{c}\text { Alert } \\
\text { Type }\end{array}$ \\
\hline 1 & 17.5 .7 .8 & 21 & 22 & 6 & $5 \mathrm{~ms}$ & Low \\
\hline 2 & 17.5 .7 .8 & 21 & 22 & 5 & $4 \mathrm{~ms}$ & Low \\
\hline 3 & 17.7 .7 .8 & 21 & 22 & 8 & $7 \mathrm{~ms}$ & High \\
\hline 4 & 17.7 .7 .8 & 44 & 46 & 11 & $5 \mathrm{~ms}$ & Low \\
\hline 5 & 17.7 .7 .8 & 44 & 48 & 18 & $4 \mathrm{~ms}$ & Low \\
\hline 6 & 20.5 .5 .7 & 44 & 45 & 5 & $8 \mathrm{~ms}$ & High \\
\hline 7 & 20.5 .5 .7 & 46 & 49 & 11 & $7 \mathrm{~ms}$ & Low \\
\hline 8 & 23.6 .6 .9 & 48 & 49 & 12 & $6 \mathrm{~ms}$ & High \\
\hline 9 & 23.6 .6 .9 & 48 & 49 & 13 & $8 \mathrm{~ms}$ & High \\
\hline
\end{tabular}

\section{Ddos Attack Prevention In An M-healthcare System}

The DDoS attack creates major trouble in the m-healthcare system by flooding the target network with the repeated request for the same data. This will prevent the user to access the sensitive data on time and as a result, the diagnosis of a patient, report generation, communication between internetworking systems is delayed. The following section will analyze the DDoS attack prevention mechanism.

\section{The following steps (Step 1 to Step 8) will be useful to prevent a DDoS attack}

Step 1:The M-health system response plan should be ready in case of security breaches and respond as promptly as possible.

Step 2:The plan should include methodologies on how to make the m-health system activities operational in case of DDoS attack is successful.

Step 3:Technical competencies and expertise will also be necessary and a proper system checklist to be introduced to get an idea of advanced threat detection.

Step 4:Passing information among the stakeholders involved in the m-healthcare system is important. 
Step 5:Equip the network, applications, and infrastructure with multilevel protection strategies that are highly desirable. This may include firewalls, VPN, anti-spam, content filtering, etc.

Step 6:Cloud providers also provide cloud-based solutions. The cloud providers implement security protocols both at the virtual and physical levels.

Step 7:Vulnerability assessment of the network is mandatory. A vulnerability assessment involves identifying security exposures so that infrastructure can be better prepared for DDoS attacks. This is done by taking account of all devices on the network, system information, any vulnerability associated with them, and the up-gradation of the system for further assessment.

Step 8:Identify the warning signals of DDoS attacks like Slow connection, intermittent web crashes.

\subsection{Technical Competency for DDoS attack prevention}

Technical competency is described by the application-specific skills and bits of knowledge to perform a certain job efficiently. Technical competency is indispensable in preventing DDoS attacks in the system. The infrastructure and people must be trained to detect the attack. The authors have identified few attributes which are helpful to prevent the attack in the system. The attribute specification is explained properly.

$T_{1}$ : System administrators must have sufficient knowledge in helping application servers containing sensitive medical data.

$T_{2}$ : The architecture of the mobile healthcare system knowledge through which data is passed across the network.

$T_{3}$ :System administrators must have sufficient knowledge in handling cloud-loT-based systems.

$T_{4}$ : The administrator must have sufficient knowledge about the normal network traffic.

Technical competency is denoted by $\mathrm{T}_{\mathrm{C}}$

$$
T_{C}=\bigcup_{i=1}^{n} T_{C_{i}}(1)
$$

Where ' $n$ ' is the independent technical competency.

\subsection{DDoS attack information exchange through message passing system}

The information exchange between different nodes in the system is very important when attack traffic reaches above the normal level. The node will not able to execute its processes if it is affected by a DDoS attack. The node can internally pass the information to the neighboring node or it can broadcast the message. The exchange of attack information has a wide level of significance. The remaining nodes in the system can be alert and take immediate measures to tackle the attack. The affected node can transfer the suspended execution of the process to the nearest neighboring node. The Algorithm [2] of Fig. [5] describes the passing of messages to the different nodes in the system.

The Fig. 6. indicates complete broadcast information for the internetworking system to specify the message regarding the DDoS attack that happened in the system and it is rooted at a particular node $\mathrm{P}_{\mathrm{r}}$. The entire scenario is considered based on the flooding algorithm. The message passing starts from $\mathrm{P}_{\mathrm{r}}$ and then it sends its message to all the neighbors that are under the networking communication path. Node $\mathrm{P}_{\mathrm{i}}$ receives the message for the first time and from the nearest node ((say $\left.P_{j}\right)$ ) it will circulate the message to all the neighboring nodes except $P_{j}$. 
Theorem 1: The execution of the broadcast algorithm in the synchronous model, all the nodes situated at distance $\mathrm{x}$ concerning $P_{r}$ in the spanning tree bound to receive message $M_{S}$ in the round of $x$.

Proof: It is assumed that a node is situated at distance $x$ from $\mathrm{P}_{\mathrm{r}}$. The proof of the algorithm can proceed based on induction. If the $x=1$, then the theorem indicates each of the child nodes of $P_{r}$ receives $M_{S}$ in the first round. Every node is situated at a distance from $P_{r}$ receives the message $M_{s}$ in the round of $x-1$. In the same way, every node $P_{k}$ which is at distance $x$ from $P_{r}$ in the networking system receives $M_{s}$ in round t. Let $P_{i}$ be the parent of $P_{k}$ in the message passing system. $P_{i}$ is located at a distance $x-1$ from $P r$, so based on induction $P_{j}$ will receive $M_{s}$ in the round of $x-1$. $P_{j}$ will send the message to $P_{k}$ in the next round.

Theorem 2: Every execution in the asynchronous model of message broadcasting system finds to construct a tree whose root is at $P_{r}$. and covers all the nodes of the internetworking system.

Proof The proof of the algorithm is constructed based on the flooding algorithm. Firstly, the node will set its parent variable and this will never be changed. It is assumed that the value of the children variable can never be decreased. As a result, the graph structure created by the parent and child variables is static. Subsequently, the parent and children variables at different nodes are the same i.e., if $\mathrm{P}_{\mathrm{i}}$ is the child of $\mathrm{P}_{\mathrm{k}}$, then $\mathrm{P}_{\mathrm{k}}$ is the parent of $\mathrm{P}_{\mathrm{i}}$. It is observed that the resulting graph maintains the properties of a spanning tree whose root is at $P_{r}$. The construction of a spanning tree increases the message complexity by a constant multiplicative factor. The asynchronous model indicates that the message $\mathrm{M}_{\mathrm{s}}$ reaches all the nodes that are at distance $x$ from $\mathrm{P}_{\mathrm{r}}$.

\subsection{DDoS attack prevention algorithm}

The following section demonstrates the DDoS attack prevention algorithms. The algorithms are subdivided into net flow calculation, Net flow separation algorithm, and the marking algorithm. The Algorithm [3] of Fig. [7] calculates the net flow of the received requests from each of the client and send the response to the network monitor. The algorithm will check the received request for each time. It is observed that if the received request is almost equal to the net average flow of the system, then attack details will be sent to the network monitor. In a particular timeframe if the received request reaches $>2 *$ average network flow, then an alert message will be triggered and details will be sent to the network monitor.

The Net Flow Separation Algorithm [4] of Fig. [8] specifies the types of DDoS attack happened in the m-healthcare system. The algorithm majorly specifies the three types of attack states which are indicated by High level, Middle Level, and lowlevel attack. The attack scenario will be different based on changing combination of attack source and destination port individually. The following part presents the complete description of the different attacks in the system.

\subsubsection{High-level attack:}

The high-level attack can intercept the encrypted message and forward the message to the target destination. This will inject false information to the different nodes in the $m$-health system. The effect of this false information injection may hamper the privacy and security of the whole system. The major objective of this attack is to overload the target server and steal sensitive information by creating a duplicate identity of the legitimate user. This type of attack is very difficult to predict and uses a man-in-the middle-attack technique to access the confidential server resources.

\subsubsection{Middle-level attack:}

The objective of the middle-level attack is also to penetrate the system in an unauthorized way and break the confidentiality and privacy of the whole system. The functionality of the attack is different. Here all the malware agents are not active at a time. Few are active in a particular iteration and the other remaining will send requests to the next attacking moment. All malware agents are used to receive a unique identification (ID) number. The iteration number of sending requests is always changing. The odd-numbered agents will send the request to the odd iteration number. The system forces to send even- 
numbered agents to send requests for the even number iteration. It is observed that if $\mathbf{3 0}$ iterations are there in a system with a fixed time frame of 5 , then at iteration 5 all the odd-numbered agents will attack the server. The even-numbered will be initiated for the next iteration number 10 .

\subsubsection{Low-level attack:}

In this attack, all the attacker agents make complete coordination with each other and perform the attack at a fixed interval. The attacking moment is dependent on the number of iteration and fixed time intervals. For instance, if 50 iterations are there and it is divided into 10 fixed time intervals then the attacker agents will attack 5 times in the system. The low-level attack is initiated by checking the remainder of the iteration number and fixed time interval. During this checking, if the value becomes zero, then the attack moment will be initiated. otherwise, the malware agents have to wait for the next attacking moment.

The attack prevention mechanism is also a very important and challenging technique. The authors have considered two DDoS preventive mechanisms. They are named Frequency Prevention (FP) and Coordinated Prevention (CP) approach. A detailed description of the approaches is given below.

\subsubsection{Frequency Prevention (FP):}

The frequency prevention technique is used to determine whether a service will be provided to the agent or it is denied. The technique follows a strategy to check the origin of the request. For instance, if the request comes from a malware agent it will not directly provide the service. The frequency of the agent is calculated as follows.

$f p_{t}(\mathrm{x})=f p(x) /\left(C_{n}+1\right)(2)$

where $\mathrm{fp}_{\mathrm{t}}(\mathrm{x})$ is the frequency of received request per unit time, $f p(x)$ determines the absolute frequency of the source $x$ and $\mathrm{C}_{\mathrm{n}}+1$ determines the number of iterations. Finally, if the frequency per time unit is lower than the threshold value then service will be provided. Otherwise, it will be discarded from the list.

\subsubsection{Coordinated prevention approach (CP):}

The CP has the main aim to detect whether the attack follows any pattern or not. It is calculated as

$R_{t}=S_{\text {max }} R_{r}(3)$

where $R_{t}$ is the request threshold, $S_{\text {max }}$ is the maximum number of services per iteration and $R_{t}$ is the ratio of threshold request.

Here, each time a request is received for service and CP checks the status of the request in terms of attack perspective. If no attack history is found then grant the service immediately. For instance, if it is attacked previously then CP computes the ratio of request in attack and finds the threshold frequency of that attack. It is observed that if the ratio request is greater than the threshold then CP denies the service.

The Algorithm [5] of Fig. [9] presents the internal functionality of the system when the attacks are initiated and detected. The designing of the algorithm is completely dependent on the marking of requests through coloring. The proposed algorithm considers three colors: Red, Green, and Yellow. If the request type is green then service is instantly granted. Yellow listed request types must have to wait for further confirmation and red-listed are discarded from the list. For instance, if the request type is in the yellow list, then service is not granted instantly. The algorithm will compare the destination address with the affected address and consider the active status of the request (high or low). If the destination and affected addresses are the same then no service will be provided and blocked the request permanently. 
The Algorithms [3-5] must have a wide level of significance in the $m$-healthcare system. The algorithms will be beneficial to categorize the different types of DDoS attacks in the system. Accordingly, the automated intelligent m-health system will maintain the history of various request types to the internal database system. If the same request is initiated next time, then the status of the request can be checked through the database system, and on that basis, the decision will be taken in less time. This will help to prevent the attacks and accessing of sensitive data without proper authorization.

\section{Analysis Of Results \& Discussion}

The section presents the complete analysis of DDoS attacks in the m-healthcare system. The effect of different kinds of DDoS attacks based on their preventive approaches is completely evaluated in this section.

\subsection{Comparison of different DDoS attack-based tools}

The authors have identified different tools and standards which allows a better simulation environment for experimenting DDoS attack in the system architecture. The comparisons are based on the key features of the tools like availability, use of programming language, operating system, performance, and area of the application model. The tools are also categorized based on data Input format and Output format. Table [2] demonstrates the comparison of different tools and standards.

Table 2

Comparison of different DDoS attack-based tools

\begin{tabular}{|c|c|c|c|c|c|c|c|c|}
\hline $\begin{array}{l}\text { Sl. } \\
\text { No. }\end{array}$ & Tools & Availability & $\begin{array}{l}\text { Programming } \\
\text { language }\end{array}$ & O.S. & $\begin{array}{l}\text { Output } \\
\text { Format }\end{array}$ & $\begin{array}{l}\text { Input } \\
\text { Format }\end{array}$ & Performance & $\begin{array}{l}\text { Application } \\
\text { Model }\end{array}$ \\
\hline 1 & Cloud Sim & $\begin{array}{l}\text { Open- } \\
\text { source }\end{array}$ & Java & Windows & XML & $\begin{array}{l}\text { Command } \\
\text { Prompt }\end{array}$ & Reliable & $\begin{array}{l}\text { Data } \\
\text { Transfer, } \\
\text { Computation }\end{array}$ \\
\hline 2 & $\begin{array}{l}\text { Cloud } \\
\text { Analyst }\end{array}$ & $\begin{array}{l}\text { Open } \\
\text { Source }\end{array}$ & C++, Python & Windows & JSON & $\begin{array}{l}\text { Command } \\
\text { Prompt }\end{array}$ & Reliable & Computation \\
\hline 3 & $\begin{array}{l}\text { Cloud } \\
\text { Reports }\end{array}$ & $\begin{array}{l}\text { Open } \\
\text { Source }\end{array}$ & Java & Linux & $\mathrm{XML}$ & GUI & Consistent & $\begin{array}{l}\text { Data } \\
\text { Transfer, } \\
\text { Execution } \\
\text { Time }\end{array}$ \\
\hline 4 & $\begin{array}{l}\text { Breaking } \\
\text { Point }\end{array}$ & Proprietary & C\# & Linux & CSV & GUI & Consistent & $\begin{array}{l}\text { Data } \\
\text { Transfer, } \\
\text { Execution } \\
\text { Time }\end{array}$ \\
\hline 5 & Comsec & $\begin{array}{l}\text { Open } \\
\text { Source }\end{array}$ & Java & $\begin{array}{l}\text { Windows } \\
+ \text { Linux }\end{array}$ & $\mathrm{XML}$ & GUI & $\begin{array}{l}\text { Highly } \\
\text { reliable }\end{array}$ & $\begin{array}{l}\text { Denial of } \\
\text { service }\end{array}$ \\
\hline 6 & LOIC & $\begin{array}{l}\text { Open } \\
\text { Source }\end{array}$ & C\# & Windows & JSON & GUI & Reliable & $\begin{array}{l}\text { DOS, } \\
\text { cybersecurity }\end{array}$ \\
\hline 7 & $\mathrm{HOIC}$ & $\begin{array}{l}\text { Open } \\
\text { Source }\end{array}$ & $\mathrm{C}++$ & Linux & $\begin{array}{l}\text { JSON, } \\
\text { XML }\end{array}$ & GUI & Real-Time & $\begin{array}{l}\text { Dos Cyber } \\
\text { Security }\end{array}$ \\
\hline 8 & HULK & Proprietary & Python & Windows & $\mathrm{XML}$ & GUI & Real-time & $\begin{array}{l}\text { Denial of } \\
\text { service }\end{array}$ \\
\hline 9 & DDoSSim & Proprietary & Java & $\begin{array}{l}\text { Linux }+ \\
\text { Windows }\end{array}$ & $\mathrm{XML}$ & GUI & Bulk Value & $\begin{array}{l}\text { Denial of } \\
\text { service }\end{array}$ \\
\hline 10 & Rudy & $\begin{array}{l}\text { Open } \\
\text { Source }\end{array}$ & Python & Linux & $\begin{array}{l}\text { XML, } \\
\text { JSON }\end{array}$ & GUI & Reliable & Data Security \\
\hline 11 & GoldenEye & $\begin{array}{l}\text { Open } \\
\text { Source }\end{array}$ & Python & Linux & $\mathrm{XML}$ & GUI & Reliable & $\begin{array}{l}\text { Dos, } \\
\text { Cybersecurity }\end{array}$ \\
\hline
\end{tabular}




\subsection{Schematic diagram of DDoS attack}

The spreading of malware starts from one attacker node and tries to connect servers on the cloud network to destroy patient runtime data. If any of the nodes in the network is affected then automatically other nodes may be affected and published as an infected node. A flooding attack is initiated from at least one infected node and moves towards at least one server in the cloud network. Hence the servers are in a compromised state to enable their services to the specified nodes. The authors have proposed the DDoS preventive algorithms to maintain the server in a non-compromising state. As a result, the stakeholders can access the run-time medical data of the patient at any point of time from the specified node.

A cloud network system consists of numerous servers and access points/nodes. They often have different levels of vulnerabilities from cyber-attack. Most nodes are vulnerable to flooding and malware attacks but the servers are protected from malware as they are commonly behind the firewalls. Fig. [10] presents a complete schematic diagram of a DDoS attack in a cloud-based environment.

\subsection{Attack Configuration}

The simulation tool should be configured in such a way that it can accommodate from simple attacks to flooding attacks. The target server will be attacked by a large number of packets and observe the effects during the simulation process. So, the authors have divided the experiment based on low-level attack, medium level attack, and high-level attack. The experiment is simulated using Cloud Sim 3.0, so it is possible to multiply the number of CPU, VM, peer nodes, storage devices, and other combinations.

\subsubsection{Attack volume}

The experiment is started with five nodes with a certain number of VMS (7CPU x64, 64 bit, $4.4 \mathrm{GHz}, 8 \mathrm{~GB}$ of physical memory, 1TB of cloud storage, 128bit SSL security) and 50000 base 64 encoded packets per second. The duration can vary from 600 seconds to 43200 seconds. The default value is set to 7200 seconds and increases gradually. The bandwidth is considered from $300 \mathrm{~KB} /$ second to $10 \mathrm{MB} /$ second. To achieve a small evaluation time, the authors have started the attack period $\mathrm{T}=120 \mathrm{~s}$ with the auto-scaling mechanism. The environment supports the incrementing of the other five VMS for three hours of attack to get maximum service degradation.

\subsubsection{Attack Pattern}

The research analyses the traffic data from cloud networks where the cloud provider host supports more than 10000 services including web service, mobile application, data analytic form on patient medical data. The authors have observed the net flow logs from the cloud provider for a couple of days and found various protocol numbers, TCP flags, and port numbers. The following protocols are included like UDP, mail, SSH, TCP flag like TCP SYN flood, UDP flood, port scan. Port numbers play a significant role to filter traffic. TCP traffic with the destination port number 3306 is used to filter the SQL traffic.

The research focuses on multidimensional attacks which can be performed on network, transport, and application layer. The authors have considered four parameters like Source IP, Packet/Second, Destination port, and delay to monitor the attack pattern. During the simulation process, one parameter is restricted as constant and iterates other parameters with different values. The process continues for the remaining parameters also. The following Table [3] provides a detailed description of different network-based attacks in a cloud environment. 
Table 3

Summary of different network-based attacks

\begin{tabular}{|c|c|c|c|c|c|}
\hline & Attack type & $\begin{array}{l}\text { Target } \\
\text { Resource }\end{array}$ & Detection Type & Delay(minute) & Attack Description \\
\hline 1. & SQL Injection & SQL Server & Spread based & 25 & $\begin{array}{l}\text { SQL queries are sent to exploit a software } \\
\text { vulnerability }\end{array}$ \\
\hline 2. & Brute-force & $\begin{array}{l}\text { Server } \\
\text { Resources }\end{array}$ & Spread based & 60 & Break the weak password for server contro \\
\hline 3. & Port Scan & $\begin{array}{l}\text { Server } \\
\text { Resources }\end{array}$ & Signature-based & 60 & $\begin{array}{l}\text { Scan for open ports for administrative } \\
\text { control }\end{array}$ \\
\hline 4. & $\begin{array}{l}\text { Malicious } \\
\text { web activity }\end{array}$ & Users & $\begin{array}{l}\text { Pattern of } \\
\text { communication }\end{array}$ & 120 & $\begin{array}{l}\text { Communicate with malicious agents to } \\
\text { destroy privacy }\end{array}$ \\
\hline 5. & UDP flood & $\begin{array}{l}\text { Network } \\
\text { bandwidth }\end{array}$ & Volume-based & 1 & Send the packets to a fixed or random port \\
\hline 6. & $\begin{array}{l}\text { TCP SYN } \\
\text { flood }\end{array}$ & $\begin{array}{l}\text { Server } \\
\text { Resources }\end{array}$ & Volume-based & 2 & Send the packets to a fixed or random port \\
\hline 7. & DNS attack & $\begin{array}{l}\text { Network } \\
\text { bandwidth }\end{array}$ & Volume-based & 30 & $\begin{array}{l}\text { A huge request is generated by malware } \\
\text { agents to overload the DNS server }\end{array}$ \\
\hline
\end{tabular}

\subsubsection{Attack Traffic}

Attack traffic identification is one of the major challenging areas in a cloud-based attacking environment. The authors have presented the attack traffic specification with the following fields: Delay (in millisecond), Destination Port, Packet received /second from the different clients, and Source IP. The following table demonstrates the attack traffic generated during the simulation-based experiment environment.

Table 4

Attack traffic specification

\begin{tabular}{|c|c|c|c|c|}
\hline & Delay & Destination Port & Packet/Second & Source IP \\
\hline 1. & 40 & 46 & 50000 & 17.7.7.8 * \\
\hline 2. & 55 & 46 & 62000 & 17.7.7.8 * \\
\hline 3. & 72 & 46 & 75000 & 17.7.7.8 * \\
\hline 4. & 86 & 46 & 80000 & 17.7.7.8 \\
\hline 5. & 120 & 46 & 88000 & 17.7.7.8 \\
\hline 6. & 150 & 46 & 94000 & 17.7.7.8 \\
\hline
\end{tabular}

\subsubsection{Attack Simulation Process}

In the attack simulation process on each cloudlet, the authors have run the following commands

hping3 -I u40 -s -p 80 -c 50000 17.7.7.8

s -> It specifies sending SYN packets

$-\mathrm{P}->80$ targets port 80 
-I -> U40 waits 40 microseconds between packets $=50000$ packets per second and the following output received from the simulation process.

The process will continue with the repeated interval of two to three minutes. The authors have selected the cloudlet, unbind the port and change the rate of packets by modifying the $-i$ ux flag where $x$ will increase/decrease by 5 . This will change the packets/second in each iteration. During the runtime simulation, the whole situation is observed and experience the following things.

- Transaction latency

- the loading time of page

- completed number of transactions

- Number of resumed transactions

- The number of halted transactions.

\subsubsection{Experimental Setup}

The system architecture is specified by making a virtualized cloud-based environment that satisfies the all-essential conditions of a DDoS attack. The complete execution environment is comprised of three layers. The uppermost layer is connected with the $10^{6} \mathrm{VM}$ (Virtual Machine) and each of the VM memory is $1024 \mathrm{~KB}$ in dual-core machine configuration. The experiment considers 5 cloudlets are running continuously in the system environment. The next layer, where the most generalized and extensible framework-based cloud simulation software is running in the system. This is used to coordinate between the data centers, VMs, users, applications, and computational resources. The application layer consists of the various attacker and authentic VMS which are running in windows and Linux-based operating systems. The execution environment considers 50 honest agents and 25 malware agents to experiment. The authors have considered a maximum of 25 hours to predict the changing scenario of different attacks in the system.

The major attacks are executed on UDP, TCP, and BGP protocols, and a protocol-based packet sniffer is used to filter the data packets as set by the network intelligence system. The acceptance and discarding of packets are completely decided by the rules framed by the networking system. The DDoS attacks are simulated to send a large volume of data packets for high bandwidth and resource consumption. The intelligent system will receive the corresponding IP addresses and maintain a log file for future analysis of the data set.

\subsubsection{Analysis of simulated execution of DDoS attack prevention mechanism}

The evolution of attacks and the corresponding preventive strategies are experimented and analyzed with the help cloudbased simulation environment. The authors have evaluated the performance of the DDoS detection and prevention strategy concerning the time frame.

Fig. [12] presents the simulated execution of a low-level attack which is prevented by FP strategy. The major vulnerability issues of FP strategies can be exploited by low-level attacks. The attack can increase the request frequency by increasing the attack frequency. In this attack, it is observed that the failure rate is high. The number of services per iteration is much less than the number of attackers. As a result, all the attackers are not entitled to get the service.

The success of the DDoS attack is completely dependent on the success of the clients. The frequency of attack and the success of the clients are not proportional to each other. The sending of successful client requests will become zero at the time of execution of a DDoS attack in the system. The legitimate client's request will not be forwarded and the performance of the system will be degraded. The probability of a DDoS attack is low as most of the attackers are not able to get service from the server agent due to an insufficient number of services. 
Fig. [13] depicts the simulated execution of the low-level attack based on CP strategy. In the first iteration of the second hour, the success of the client is very low as the attackers are not detected properly. The honest agents and the malware agents are not tracked properly. The problem of such identification leads to get success of DDoS attacks in the system. As the simulation continues concerning time frame the honest agents are identified. The misleading agents are segregated from the list. At the 20th hour, the success of the attackers becomes low and the success of the client request goes high. It is observed that at any point in time client success may fall to zero due to the unavailability of requests from honest agents. This will generate an exceptional situation in the simulated environment.

Fig. [14] shows the simulated execution of middle-level DDoS which is prevented by CP strategy. The middle-level attack is difficult to track as compared to low-level attacks since the attacks are always performed by different agents. So, the identification of honest agents and malware agents is very difficult. DDoS attacks are successful in most of the attack cases and the success of the client is reduced to zero. The attacks which are initiated by the outside of the network play a major role to make the DDoS attack successful. As per the strategy of the middle-level attack, all the agents are not active at a time. So, there is less chance of denial of a request by both honest and malware agents.

Fig. [15] shows the simulated execution of middle-level attacks along with the FP strategy. The preventive mechanism based on frequency is much more suitable as it considers all the iterations during the attack period. It is not dependent on a particular iteration where the attacks are initiated successfully. Here DDoS attack is successful in most cases and the success of the client request reaches zero. This will lead to the rejection of maximum requests by honest agents.

Fig. [16] and Fig. [17] show the simulated execution of high-level DDoS attacks along with CP and FP strategies. The highlevel attack is very difficult to predict since most of the cases honest agents and malware agents are not properly identified. The behavior of an honest agent has much more similarities with the attacker. This will lead to the success of the DDoS attack by decreasing the value of successful client requests to zero or nearer to zero.

The result section is progressed with the help of two DDoS preventive mechanisms (FP \&CP). The advantage of FP over CP is its large dynamic range and constant relative accuracy. After several cycles of processing, the maximum success score and average success score are presented for a time frame. The paper discusses the DDoS attack detection and prevention mechanism. The high-level attack is chosen with best-case and the model with a middle-level attack is considered in the average case. The paper explores the range of time with the precision of data filtering to determine the optimum level of accuracy. Fig. $[18,19]$ indicates the attack success is changing with time. As soon as the attack is initiated it will not be able to penetrate the system. The prevention techniques will restrict the attacker and unfortunately, few are passed to the system. This will certainly deviate the value of the success sore. However, there are certain cases which reflects unavoidable error and cannot be considered here. The hybridization of both the technique may provide different sets of results which are not considered as a part of this research paper.

\section{Conclusion}

In m-Health, a large number of loT-based heterogeneous devices communicate with each other to transfer the patient medical data and other sensitive information through the cloud-based internetworking system. The entire path of communication is susceptible to different types of attacks in the system. The major vulnerable attack is the DDoS attack which can destroy the privacy and accessibility of sensitive data. In this paper, we focus on m-healthcare architecture and propose a DDoS detection algorithm to easily identify the attacks in the system. The entire research work divides the DDoS attack into low-level, middle- level and high-level. The two preventive mechanisms (CP \& FP) are explained and novel prevention algorithms are designed to restrict the access of attacks in the system architecture. The cloud-based experimental setup is established to check the working functionality of the prevention schemes over the different types of attacks in the system. The maximum and average success of the attack is presented concerning time. The deviation of success rate in different cases confirms the working of the prevention algorithms over the attacks. The success of a DDoS

Page $16 / 27$ 
attack increases the failure of client requests in terms of accessing sensitive data as it works in reciprocal order. During the evaluation of the experiment, we consider optimal parameters to make our approach reliable and flexible.

For future work, we will try to improve the approach for adaptive other types of non-trivial DDoS attacks. Meanwhile, we will consider that some of the nodes could send false information and that should be recognized effectively to reduce the complexity of the system.

\section{Declarations}

Being the corresponding author, I declare that there is no conflict of interest between the authors or with any organization. The information about relevant data (if any) has been provided by the authors in the manuscript.

Kamta Nath Mishra

(Digital Signature)

\section{Data Availability Statement}

All data generated or analyzed during this study are included in this published article (and its supplementary information files).

\section{References}

1. Dayal N, Srivastava S., "Analyzing behavior of DDOS attacks to identify DDOS detection features in SDN," In 2017 9th Int Conf Commun Syst Networks, COMSNETS 2017; 2017. p. 274-81. DOI: 10.1109/COMSNETS.2017.7945387.

2. Yang L, Zhao H., "DDoS attack identification and defense using SDN based on machine learning method", In Proc. 2018 15th Int. Symp. Pervasive Syst. Algorithms Networks, I-SPAN 2018. IEEE; 2019. p. 174-8. DOI: 10.1109/ISPAN.2018.000036.

3. Priyadarshini, Rojalina, \& Barik, Rabindra Kumar (2019), "A deep learning-based intelligent framework to mitigate DDoS attack in fog environment", Journal of King Saud University Computer and Information Sciences. https://doi.org/10.1016/j. jksuci.2019.04.010

4. Krishnan, Prabhakar, Duttagupta, Subhasri, \& Achuthan, Krishnashree (2019)," VARMAN: Multi-plane security framework for software-defined networks”, Computer Communications, 148, 215-239.

5. Sharafaldin, I., Lashkari, A. H., Hakak, S., \& Ghorbani, A. A. (2019), “Developing realistically distributed denial of service (DDoS) attack dataset and taxonomy", In 2019 International Carnahan Conference on Security Technology (ICCST), Chennai, India (pp. 1-8).

6. Yan, Q., Yu, F.R., Gong, Q., Li, J., 2016, "Software-defined networking (sdn) and distributed denial of service (DDoS) attacks in cloud computing environments: A survey, some research issues, and challenges", IEEE Commun. Surveys Tutorials18 (1), pp. 602-622.

7. Yi, S., Qin, Z., Li, Q., 2015, "Security and privacy issues of fog computing: a survey", In International Conference on Wireless Algorithms, Systems, and Applications. Springer, pp. 685-695.

8. M.H. Bhuyan, E. Elmroth, "Multi-scale low-rate DDoS attack detection using the generalized total variation metric", in 17th IEEE International Conference on Machine Learning and Applications, IEEE, 2018, pp. 1040-1047, HTTP: //dx.doi.org/10.1109/ICMLA.2018.00170.

9. E. Cambiaso, G. Papaleo, G. Chiola, M. Aiello, "Designing and modeling the slow next DoS attack”, in Computational Intelligence in Security for Information Systems Conference, Springer, 2015, pp. 249-259, http://dx.doi.org/10.1007/ 978-3-319-19713-5\$422. 
10. S. Hosseini, M. Azizi, "The hybrid technique for DDoS detection with supervised learning algorithms", Comput. Netw. 158 (2019), pp.35-45, http://dx.doi.org/10.1016/j.comnet.2019.04.027.

11. A.M. Alrehan, F.A. Alhaidari, "Machine learning techniques to detect DDoS attacks on VANET system: A survey", in 2nd International Conference on Computer Applications \& Information Security, IEEE, 2019, pp. 1-6, http://dx.doi.org/10.1109/CAIS.2019.8769454.

12. H. Chen, M. Liu, F. Zhongchuan, "Using improved Hilbert-Huang transformation method to detect routing-layer reduce of quality attack in wireless sensor network", Wirel. Pers. Commun. 104 (2) (2019), pp. 595-615, http://dx.doi.org/10.1007/s11277-018-6036-3.

13. A.R. Wani, Q.P. Rana, U. Saxena, N. Pandey, Analysis and detection of DDoS attacks on cloud computing environment using machine learning techniques, in Amity International Conference on Artificial Intelligence, IEEE, 2019, pp. 870-875, http://dx.doi.org/10.1109/AICAI.2019.8701238.

14. G. Yu, T. Li, J. Wei, C. Liu, Assessment of reduction of quality attacks on mobile IP networks, in IEEE International Symposium on Parallel and Distributed Processing with Applications and IEEE International Conference on Ubiquitous Computing and Communications, IEEE, 2017, pp. 449-453, http://dx.doi.org/10.1109/ISPA/IUCC.2017.00073.

15. N. Meti, D. G. Narayan and V. P. Baligar, "Detection of distributed denial of service attacks using machine learning algorithms in software-defined networks," 2017 International Conference on Advances in Computing, Communications and Informatics (ICACCI), Udupi, 2017, pp. 1366-1371.

16. Wang, A.; Chang, W.; Chen, S.; Mohaisen, A.," A Data-Driven Study of DDoS Attacks and Their Dynamics", IEEE Trans. Dependable Secure. Comput. 2020, 17, pp. 648-661.

17. Partala, J.; Keraneny, N.; Sarestoniemi, M.; Hamalainen, M.; linatti, J.; Jamsa, T.; Reponen, J.; Seppanen, T, “Security threats against the transmission chain of a medical health monitoring system", In Proceedings of the 2013 IEEE 15 th International Conference on e-Health Networking, Applications and Services (Healthcom 2013), Lisbon, Portugal, 9-12 October 2013; pp. 243-248.

18. L. Huang, X. Chen, and X. Lai, "Network security prediction method based on Kalman filtering fusion decision entropy theory," International Journal of Security and its Applications, vol. 10, no. 12, pp. 347-358, 2016.

19. F. Holotiuk, F. Pisani, and J. Moormann, "Radicalness of blockchain: an assessment based on its impact on the payments industry," Technology Analysis and Strategic Management,vol. 31, no. 8, pp. 915-928, 2019.

20. A. Saied, R. E. Overill, and T. Radzik, "Detection of known and unknown DDoS attacks using artificial neural networks," Neurocomputing, vol. 172, pp. 385_393, Jan. 2016.

21. J. Cui, M. Wang, and Y. Luo," DDoS detection and defense mechanism based on cognitive-inspired computing in SDN," Future Gener. Comput.Syst., vol. 97, pp. 275_283, Aug. 2019.

22. W. Feng, Q. Zhang, G. Hu, and J. X. Huang, "Mining network data for intrusion detection through combining SVMs with ant colony networks," Future Gener. Comput. Syst., vol. 37, pp. 127_140, Jul. 2014.

23. K.S. Sahoo, D. Puthal, M. Tiwary, J.J. Rodrigues, B. Sahoo, R. Dash, "An early detection of low rate DDoS attack to sdn based data center networks using information distance metrics", Future Gen. Comput. Syst. 89 (2018), pp. 685-697.

24. F.Harrou, B.Bouyeddou,Y.Sun and B.Kadri,"A Method to Detect DOS and DDOS Attacks based on Generalized Likelihood Ratio Test", International Conference on Applied Smart Systems, Medea, Algeria, 24-25, November 2018.

25. R.M. Saad, M. Anbar, S. Manickam, E. Alomari, "An intelligent icmpv6 DDoS flooding-attack detection framework (v6iids) using back-propagation neural network", IETE 33 (2016), pp. 1-12.

26. Aditya Rajagopalan, Manish Jagga, Anju Kumari, Syed Taqi Ali," A DDoS Prevention Scheme for Session Resumption SEA Architecture in Healthcare loT", 3rd International Conference on "Computational Intelligence and Communication Technology (IEEE-CICl-2017)", pp.1-5.

27. S. Rahimi Moosavi, T. Nguyen Gia, A.M. Rahmani, E. Nigussie, S. Virtanen, J. Isoaho, H. Tenhuen, "SEA: A secure and efficient. authentication and authorization approach for loT based healthcare systems using Smart e-Health Gateways", 
in The 6th International Conference on Ambient Systems, Networks and Technologies. 2015, pp.452-459.

28. Prajkta Kamble, Aruna Gawade, "Digitization of Healthcare with loT and Cryptographic Encryption against DOS Attacks", International Conference on Contemporary Computing and Informatics (IC31), 2019, pp.69-73.

29. Jan Henrik Ziegeldorf, Oscar Garcia Morchon, and Klaus Wehrle: "Privacy in The Internet Of Things: Threats And Challenges", Security And Communication Networks,2013, doi:10.1002/sec.795.

30. A.-M. Rahmani, N. K. Thanigaivelan, T. N. Gia, J. Granados, B.Negas, P. Liljeberg, and H. Tenhunen, "Smart e-health gateway: bringing intelligence to Internet-of-Things-based ubiquitous healthcare systems", In Proceedings of the Annual IEEE Consumer Communications and Networking Conference. NV, USA: IEEE, January 2015.

31. S. Sivagami, D. Revathy, and L. Nithyabharathi, "Smart health care system implemented using loT", International Journal of Contemporary Research in Computer Science and Technology, vol. 2, February 2016.

32. J. Zhang, N. Xue and X. Huang, “A Secure System For Pervasive Social Network-Based Healthcare," in IEEE Access, vol. 4, pp. 9239-9250, 2016.

33. K.Nishizuka, L.Xia, J.Xia, D.Zhang, L.Fang, C.Gray, "Inter organization cooperative DDOS protection mechanism". Accessed: May. 1, 2019. [Online]. Available:Draft.https://tools.ietf.org/html/draft-nishizuka-dots-inter-domainmechanism-02.

34. Steinberger, J., Kuhnert, B., Sperotto, A., Baier, H., Pras, A.," Collaborative DDOS defense using flow-based security event information", In NOMS 2016-2016 IEEE/IFIP Network Operations and Management Symposium, pp. 516-522, April 2016.

35. K. Giotis, M. Apostolaki, and V. Maglaris. "A reputation-based collaborative schema for the mitigation of distributed attacks in sdn domains", In IEEE/IFIP Network Operations and Management Symposium, 2016.

36. Zhang, G., Parashar, M.: "Cooperative defense against DDoS attacks".J. Res. Pract. Inf. Technol. 38(1), pp. 69-84 (2006).

37. B. Rashidi, C. Fung, and E. Bertino, "A Collaborative DDoS Defence Framework Using Network Function Virtualization," in IEEE Transactions on Information Forensics and Security, vol. 12, no. 10, pp. 2483-2497, Oct. 2017.

38. Wang, A.; Chang, W.; Chen, S.; Mohaisen, A.," A Data-Driven Study of DDoS Attacks and Their Dynamics", IEEE Trans. Dependable Secure. Comput. 2020, 17, 648-661.

\section{Figures}




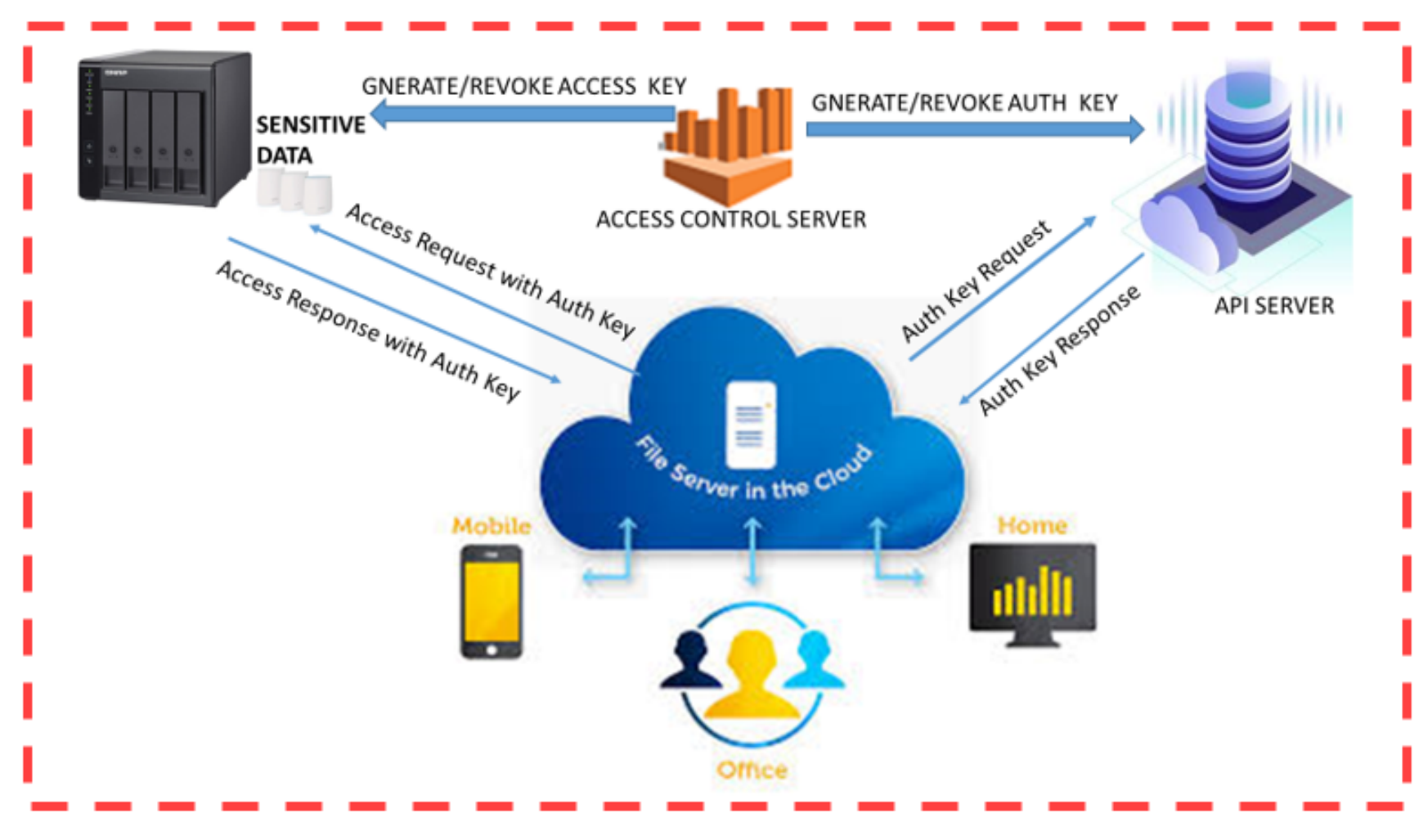

Figure 1

Basic Sensitive data access authentication technique

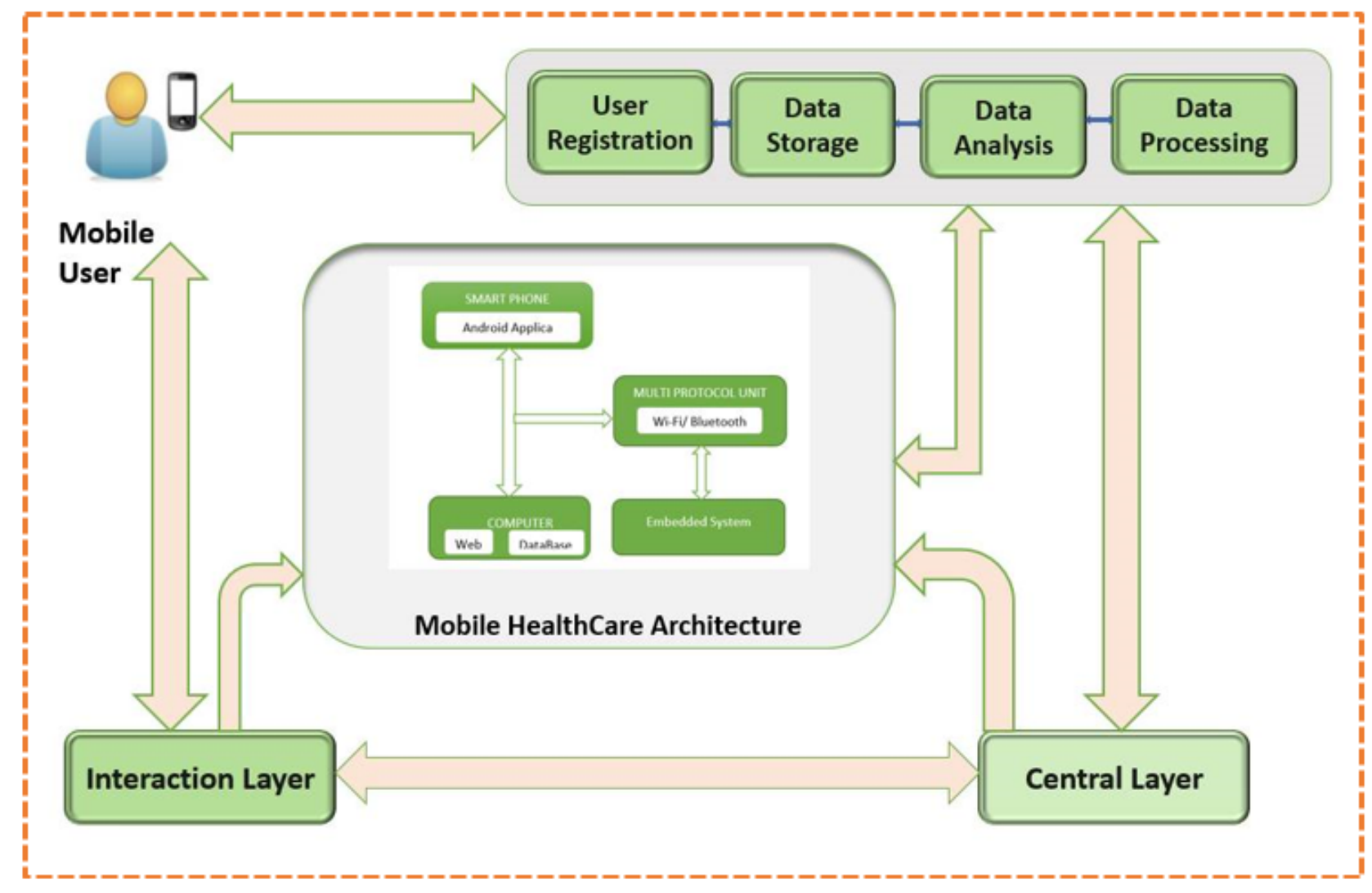

Figure 2 


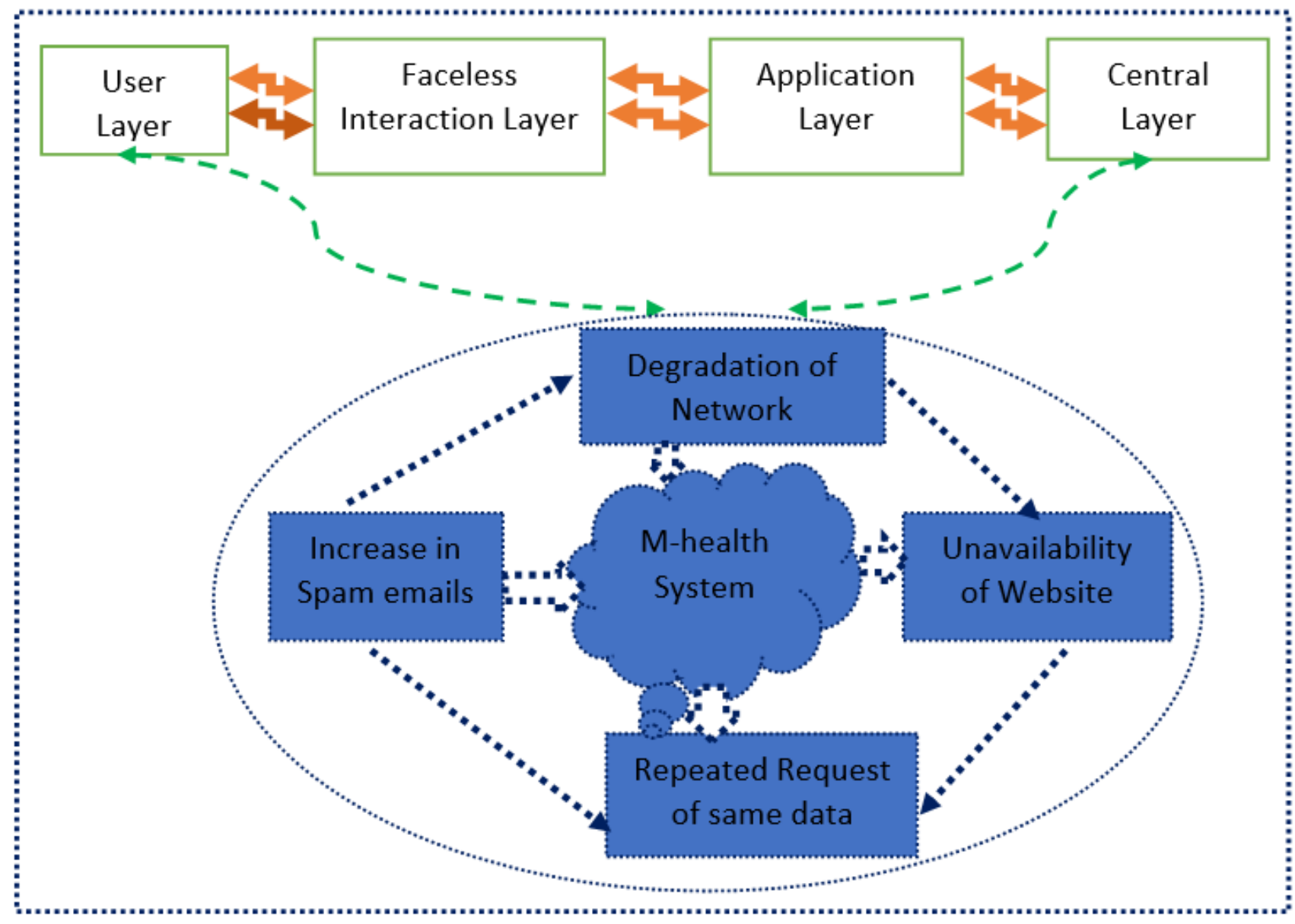

Figure 3

Layered Interaction Model of m-health system 
Input: Packets sent from the different ports at a certain time interval

Output: Detection of DDoS attack

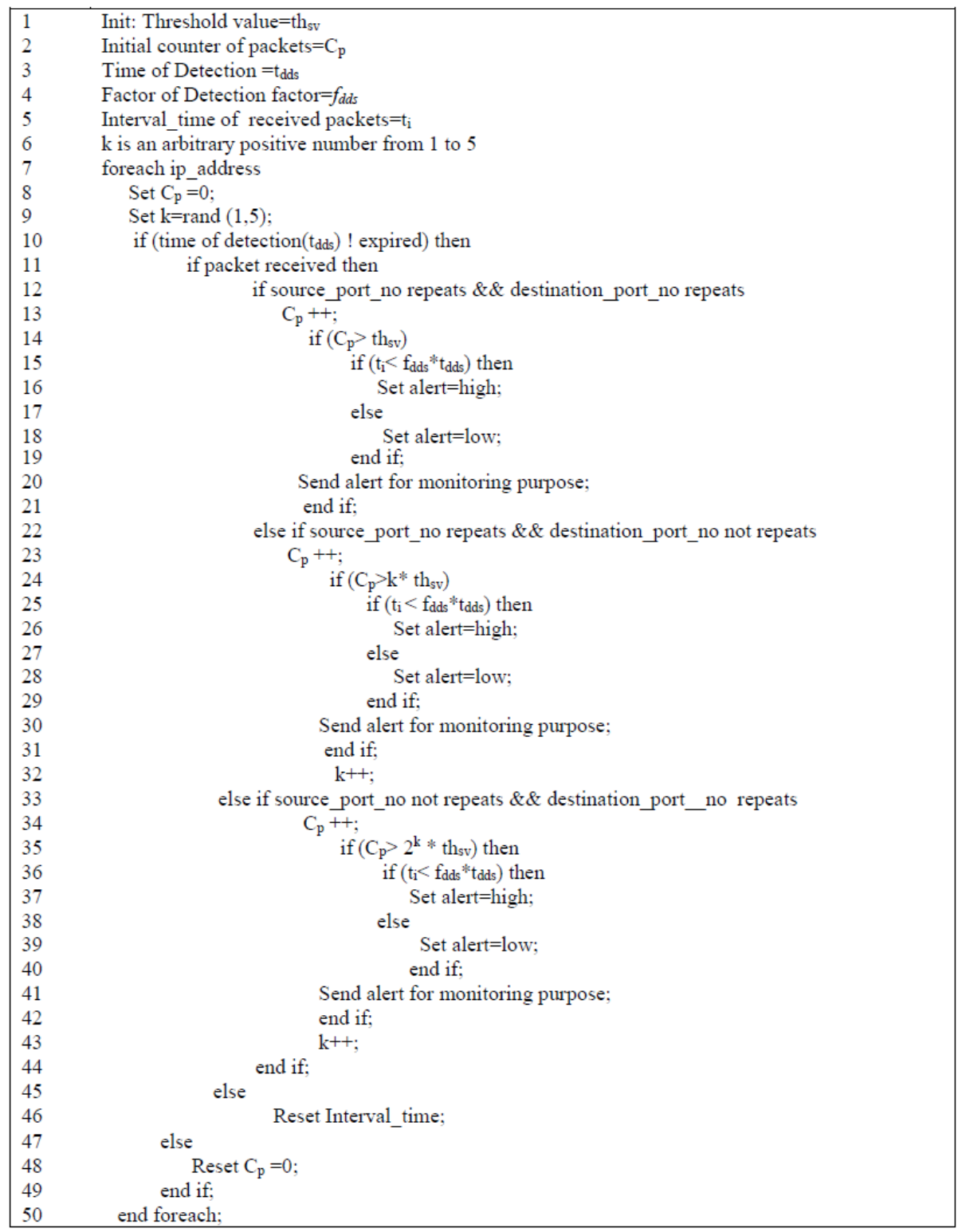

\section{Figure 4}

\section{The Proposed DDoS Detection Algorithm}




\section{Algorithm [2]: Message Passing Algorithm}

Input: Message $\mathrm{m}$ is passed to all the nodes of a tree where the rooted node is $\mathrm{p}_{\mathrm{x}}$ Output: Message is received by all the stakeholders

\begin{tabular}{|ll|}
\hline 1 & Algorithm message passing (message $\mathrm{m})$ \\
3 & Initially, $\mathrm{m}$ is in transit from $\mathrm{p}_{\mathrm{x}}$ to all its children in the spanning tree \\
4 & Define for $\mathrm{p}_{\mathrm{x}}$ : \\
5 & Upon receiving no message \\
6 & exit $(0) ;$ \\
7 & Define $\mathrm{p}_{\mathrm{i}}, 0 \leq i \leq n-1, i \neq r:$ \\
8 & Message $\mathrm{m}$ is received from its parent \\
9 & Send the message $\mathrm{m}$ to all the child nodes \\
10 & exit $(0) ;$ \\
11 & \}$/ /$ End of Algorithm
\end{tabular}

Figure 5

Message passing algorithm

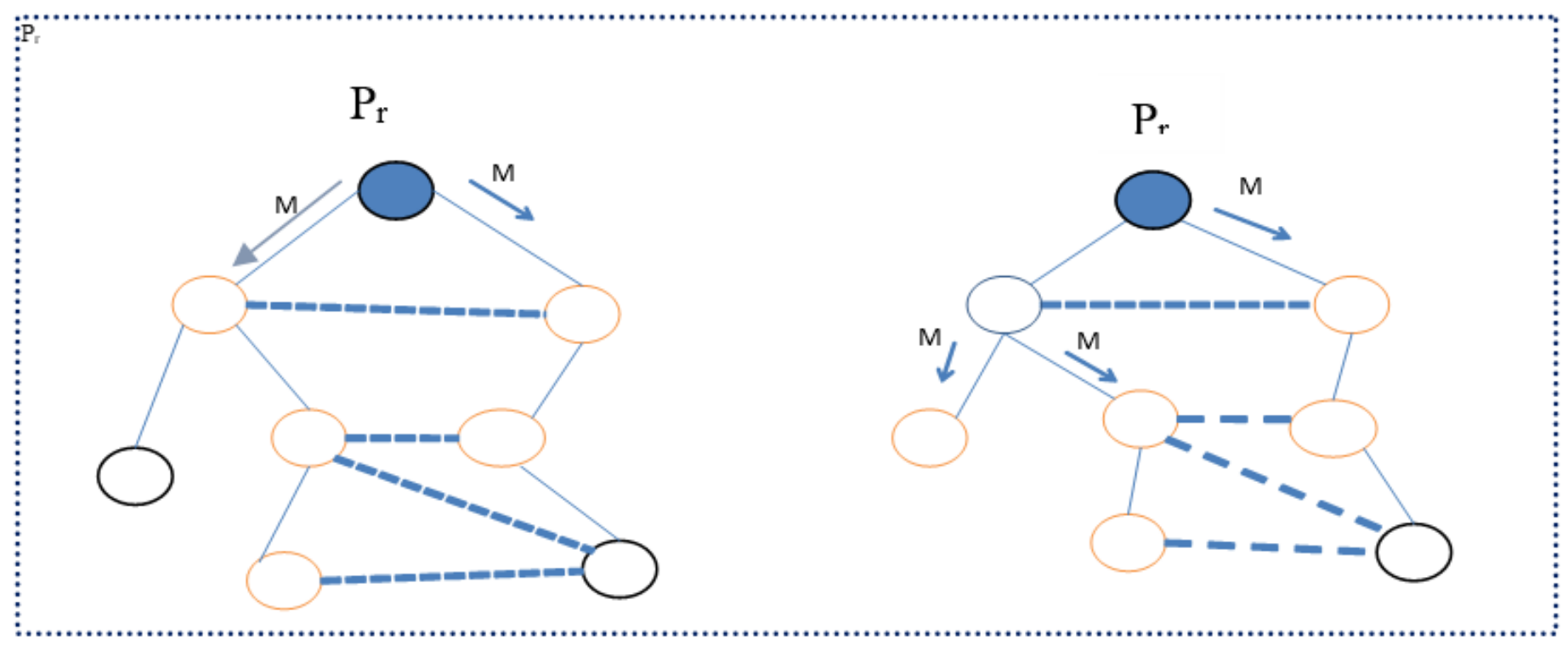

Figure 6

Two steps execution in broadcast algorithm

Figure 7

Net flow calculation algorithm 
Input: Source IP address, Source Port, Destination Port Output: Attack type identification

\begin{tabular}{|c|c|}
\hline 1 & Initialize $\mathrm{N} \leftarrow$ No of source IP addresses \\
\hline 2 & Initialize $S \leftarrow$ No of source port \\
\hline 3 & Initialize $\mathrm{D} \leftarrow$ No of the destination port \\
\hline 4 & Initialize $\mathrm{C} \leftarrow 0$; start the counter \\
\hline 5 & while $(\mathrm{N}>\mathrm{C})$ do \\
\hline 6 & for $\mathrm{i} \leftarrow 0 . . \mathrm{N}$ do \\
\hline 7 & Initialize $\mathrm{x} \leftarrow$ No of received requests from the source IP address \\
\hline 8 & for $\mathrm{j} \leftarrow 1 . . \mathrm{x}$ do \\
\hline 9 & Compare request packets with the net_avg \\
\hline 10 & if request_count $>$ net_avg \\
\hline $\begin{array}{l}11 \\
12\end{array}$ & for each $\mathrm{i}=0 . . \mathrm{N} \quad$ Set $\mathrm{S}=\mathrm{S}_{0}$; \\
\hline 13 & set $\mathrm{S}=\mathrm{S}_{\mathrm{i}}$ \\
\hline 14 & for each $\mathrm{j}=0 . . \mathrm{M}$ \\
\hline 15 & set $\mathrm{D}=\mathrm{D}_{\mathrm{i}}$ \\
\hline 16 & if $\mathrm{S}_{\mathrm{i}}$ repeats \&\& $\mathrm{D}_{\mathrm{j}}$ repeats \\
\hline 17 & Call Marking(High-level attack, N); \\
\hline 18 & else if $S_{i}$ repeats $\& \& D_{j} !$ repeats \\
\hline 19 & Call Marking(Middle-level attack, N); \\
\hline 20 & else if $S_{i}$ ! repeats \&\& $D_{j}$ repeats \\
\hline 21 & Call Marking(low-level attack, N); \\
\hline 22 & else \\
\hline 23 & Remove attack details from the list; \\
\hline 24 & Increment $\mathrm{C}$; \\
\hline
\end{tabular}

\section{Figure 8}

Net flow separation algorithm

Figure 9

Marking algorithm

Figure 10

Schematic diagram of DDoS attack 
Figure 11

\section{Attack simulation process}

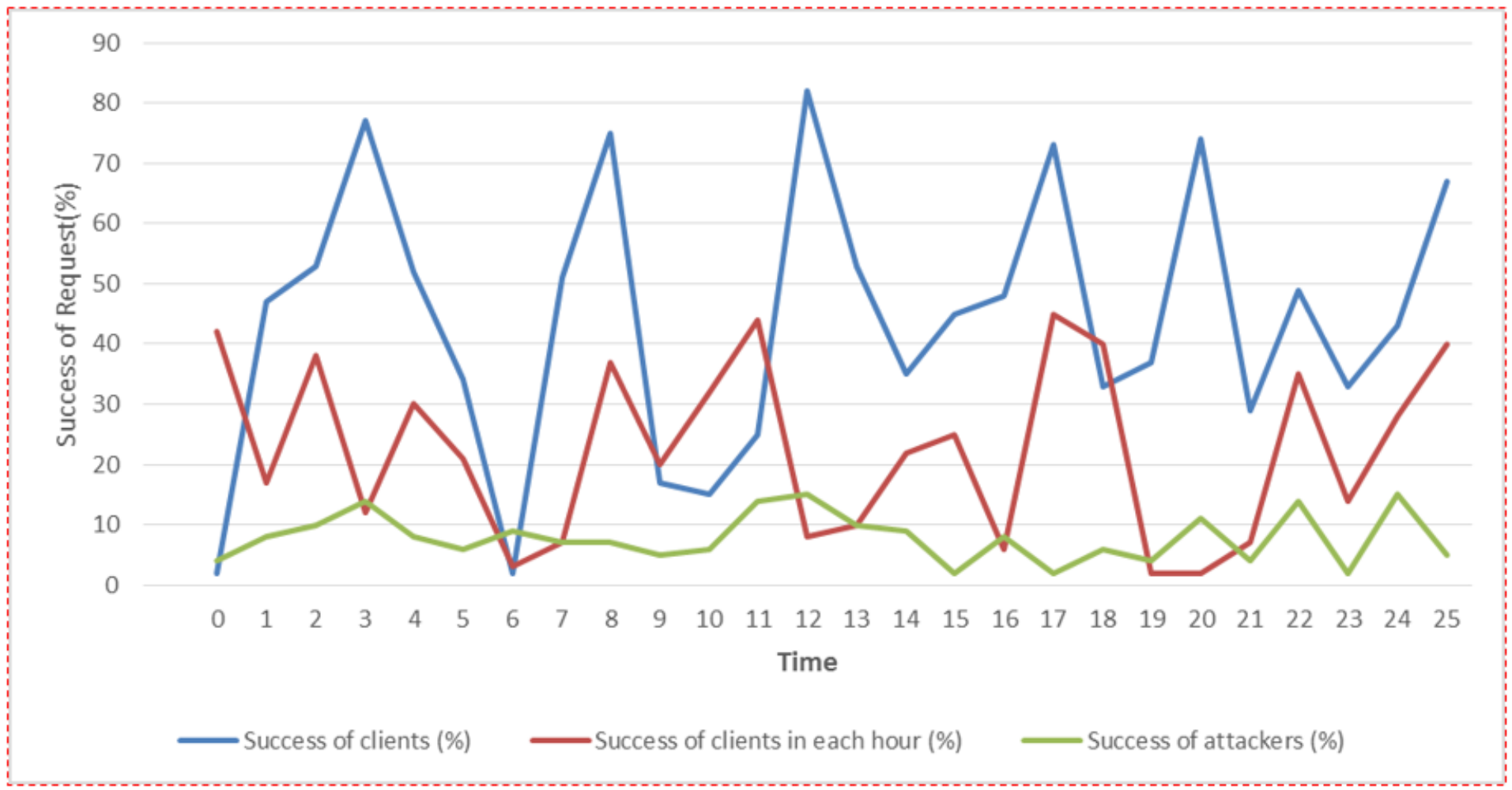

Figure 12

Frequency Prevention with the low-level attack, simulation evolution of 25hours

Figure 13

Coordinated Prevention with a low-level attack, simulation evolution of 25hours

Figure 14 
Figure 15

Frequency Prevention with a middle-level attack, simulation evolution of 25 hours

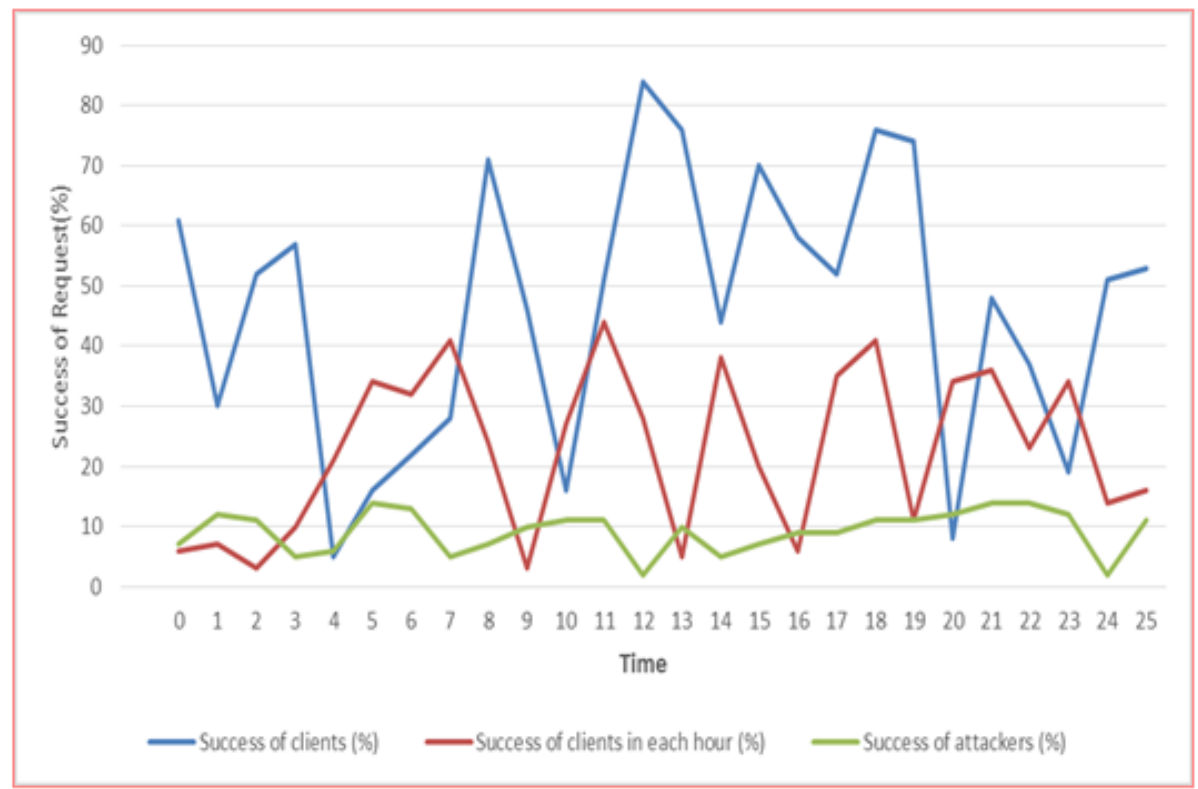

Figure 16

FP with a high-level attack, Simulation of $25 \mathrm{H}$

Figure 17

CP with a high-level attack, Simulation of $25 \mathrm{H}$

Figure 18

Maximum Success Score of DDoS attack with time

Figure 19

Average Success score of DDos attack with time

\section{Supplementary Files}

This is a list of supplementary files associated with this preprint. Click to download. 
- BiographySoumyaRayKNMSD.docx

Page 27/27 